*10. PÉREZ- CASTRO, E.; GAMALLO, G.; AVELLANA, J. A. y cols.: "Papilomas invertidos de vejiga. Presentación de tres casos y revisión de la literatura". Arch. Esp. Urol., 33: 107, 1980.

11. CALAHORRA FERNANDEZ, F. J.; USERA, G.; RELEA, F.: "Papiloma de pelvis renal asociado con carcinoma de células transicionales de vejiga: aportación de un caso y revisión de la literatura". Actas Urol. Esp., 17: 689, 1993.

12. RENFER, L.G.; KELLEY, J.; BELVILLE, W.D.: "Inverted papilloma of the urinary tract: histogenesis, recurrence and associated malignancy". J. Urol., 140: 832, 1988.

13. MULKENS, T.H.; VEREYCKEN, H.A.; VAN NUETEN, J. C. y cols.: "Bilateral ureteral inverted papillomas in association with bladder carcinoma: a case report and review of the literature". Urol. Radiol., 12: 154, 1990.

14. FRANCIS, R.R.: "Inverted papilloma in a 14 yearsold male". Br. J. Urol., 51: 327, 1979.

**15. VALERO PUERTA, J.A.; REDONDO MARTINEZ, E.; JIMENEZ GONZALEZ, C. y cols.: "Papiloma invertido transicional: expresión del antígeno nuclear Ki-67 como factor pronóstico". Arch. Esp. de Urol., 48: 887, 1995.

16. OJEA, A.: "Papiloma invertido de uretra". Act. Urol. Esp., 17: 193, 1993.

17. PELliCE, C.; CARRETERO, P.; GIL VERNET, J.M.: "Papilomas invertidos del tracto urinario inferior. Consideraciones histológicas, terapéuticas y pronósticas". Arch. Esp. Urol., 40: 165, 1987.

18. KYRIAKOS, M.; ROYCE, R.K.: "Multiple simultaneous inverted papillomas of the upper urinary tract". Cancer, 63: 368, 1989.

19. FERRERO DORIA, R.; GUZMAN MARTINEZVALLS, P.L.; MORGA EGEA, J.P. y cols.: "Inverted papilloma: presentation of 5 cases and review of the Spanish literature.” Actas Urol. Esp., 22: 131, 1998.

**20. ASANO; KOJI; MIKI y cols.: "Clinical studies the inverted papillomas of the urinary tract: report of 48 cases and rewiev of the literature". J. Urol., 170: 1209, 2003.
Casos Clínicos

Arch. Esp. Urol., 59, 2 (186-189), 2006

\section{TUMOR FIBROSO SOLITARIO DE LA TÚNICA VAGINAL.}

Marta García Torrelles, Jose Ramón Beltrán Armada, Ignacio Santolaya García, Víctor Carrascosa Lloret, Miguel Tarín Planes y Carlos San Juan De Laorden.

Servicio de Urología. Hospital Universitario Dr. Peset. Valencia. España.

Resumen.- OBJETIVO: Presentamos un caso de tumor fibroso solitario de la túnica vaginal, una lesión paratesticular de naturaleza benigna.

MÉTODO: Se llevó a cabo una exploración quirúrgica con biopsia peroperatoria, y excisión de la lesión, dejando indemnes teste y epidídimo.

RESULTADOS: la biopsia intraoperatoria reveló la presencia de tejido fibroso sin celularidad maligna. El diagnóstico histopatológico de la pieza quirúrgica fue de tumor fibroso solitario de la túnica vaginal. El paciente está asintomático y libre de enfermedad un año tras el tratamiento.

CONCLUSION: Casi todas las masas testiculares son malignas, pero hay que ser cautos ante los hallazgos ecográficos y quirúrgicos de una lesión paratesticular de probable naturaleza benigna, con el fin de evitar una orquiectomía radical no indicada.

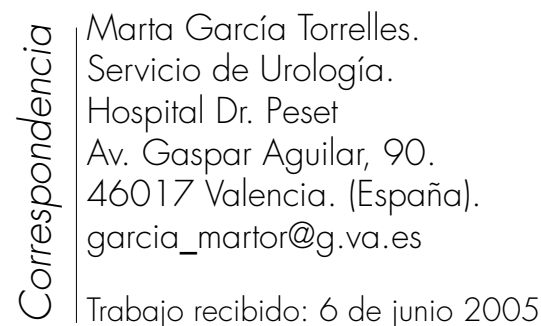


Palabras clave: Teste. Túnica vaginal. Tumor fibroso solitario. Lesión paratesticular.

Summary.- OBJECTIVES: We report one case of solitary fibrous tumor of the tunica vaginalis, a benign paratesticular lesion.

METHODS: Surgical exploration with intraoperative biopsy and excision of the lesion was undertaken, leaving the testicle and epididymis undamaged.

RESUITS: Intraoperative biopsy revealed the presence of fibrous tissue without malignant cellularity. Pathological diagnosis on the surgical specimen was solitary fibrous tumor of the tunica vaginalis. The patient remains asymptomatic and disease-free one year after treatment.

CONCLUSIONS: Almost all testicular masses are malignant, but in order to avoid a non-indicated orchyectomy, one should be cautious with the finding of a paratesticular lesion of probable benign nature on ultrasound or during surgery,

Keywords: Testis. Tunica vaginalis. Solitary fibrous tumor. Paratesticular lesion.

\section{CASO CLÍNICO}

Paciente varón de 22 años de edad, sin antecedentes de interés salvo ser fumador habitual de marihuana, acude a urgencias refiriendo aumento de tamaño de la bolsa escrotal izquierda tras un esfuerzo físico 24 horas antes, con dolor de inicio brusco y moderada intensidad. Sin fiebre ni síndrome miccional, ni irradiación del dolor. No presentaba cortejo neurovegetativo asociado, así como tampoco alteraciones del ritmo intestinal.

A la exploración física se presenta apirético y normotenso, con buena coloración de piel y mucosas. El abdomen es blando y depresible, no doloroso y sin masas. Las fosas renales se encuentran libres. El testículo derecho no presenta alteraciones mientras que en el testículo izquierdo se aprecia una tumoración de consistencia pétrea de $2-3 \mathrm{~cm}$ de diámetro, no móvil, en el polo superior del teste izquierdo, con transiluminación negativa.

Analíticamente no presentaba ningún parámetro fuera de la normalidad.

En la ecografía testicular ambos testículos muestran una ecoestructura normal con flujo-doppler conservado, evidenciando una masa sólida extratesticular, en contacto con el polo superior del teste izquierdo de $25 \mathrm{~mm}$ con vasos en su periferia.

Se ingresa para completar estudio y filiar dicha lesión.
Siguiendo el protocolo oncológico se completó el estadiaje preoperatorio con radiografía de tórax; marcadores tumorales de testículo y analítica general; y TAC abdómino-pélvico sin encontrar alteraciones patológicas en ninguna de las pruebas.

A continuación se realizó una exploración quirúrgica vía inguinal donde se apreció una masa paratesticular izquierda ligeramente abollonada con curvas suaves, nacarada y de consistencia pétrea, de fácil disección con plano de clivaje entre esta y el polo superior del testículo izquierdo y cordón espermático del mismo lado, con algunos vasos venosos en su periferia. Se tomó una biopsia peroperatoria que resultó en tejido fibroso sin de celularidad maligna. Se completó la exéresis de la lesión, dejando indemnes teste y epidídimo, evitando al paciente una orquiectomía radical.

Con un postoperatorio sin incidencias fue dado de alta a los dos días de la intervención.

El dictamen anatomopatológico de la pieza quirúrgica es de tumor fibroso solitario de la túnica vaginal (Figura 1).

En el control postoperatorio a los dos años de la intervención, el paciente se encuentra asintomático sin evidencia de recidiva y libre de enfermedad.

\section{DISCUSIÓN}

El tumor fibroso solitario de la túnica vaginal del testículo es una lesión benigna del adulto poco frecuente y de histopatogenia poco clara $(1,2,3,4)$. Aparece en la túnica vaginal, el cordón o el epidídimo, pero el testículo se encuentra siempre respetado.

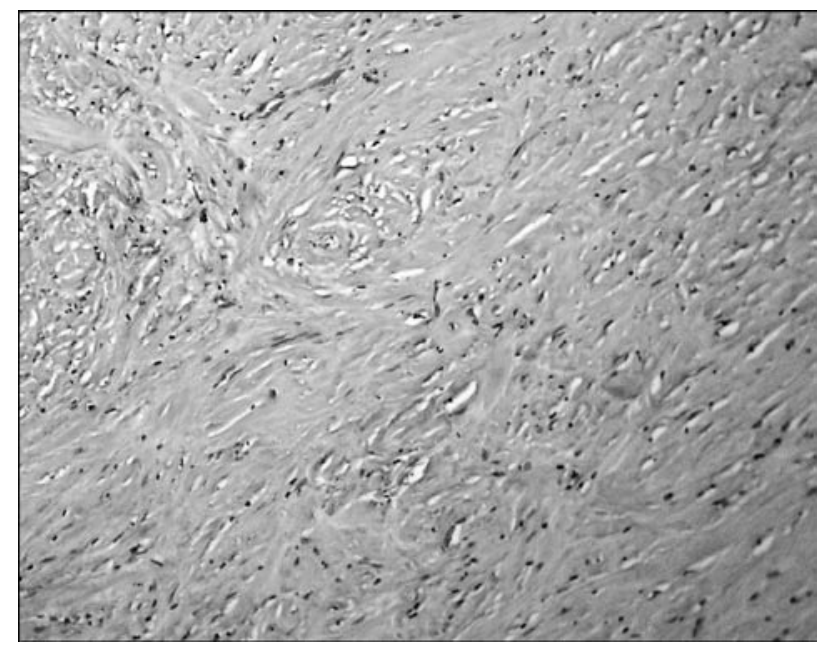

FIGURA 1. Imágen microscópica de tumor fibroso solitario de la túnica vaginal. Proliferación fibroblástica sin celularidad maligna, (HE 10x). 
Como tumor fibroso solitario se denomina habitualmente a una neoplasia característica que suele localizarse en la pleura. Ocasionalmente el tumor fibroso solitario puede aparecer en otras localizaciones, tales como pulmón, mediastino, pericardio, peritoneo, cavidad nasal y senos paranasales, y túnicas testiculares. La localización pulmonar parece la más frecuente de entre las localizaciones extrapleurales (5).

Este tipo de lesión de origen e histopatogenia muy discutidas, por su rareza y su ubicuidad además de la variabilidad en su componente inflamatorio, ha recibido diversas denominaciones como mesotelioma benigno, submesotelioma, tumor fibroso de la pleura y en su localización en la túnica vaginal testicular: fibroma, tumor benigno fibroso paratesticular, mesotelioma fibroso, funiculitis proliferativa, periorquitis fibromatosa, pseudotumor inflamatorio y pseudotumor fibroso de la túnica vaginal. Estas proliferaciones fibrosas benignas paratesticulares han sido generalmente consideradas como variantes de un pseudotumor fibroso y pocas veces como verdaderas neoplasias fibrosas de la túnica vaginal (1-5).

La primera descripción de un tumor fibroso benigno de la túnica testicular como tal se le atribuye a Astley-Cooper en $1830(1,2)$, y con gran detalle la realizada por Balloch en 1904. Cuarenta y dos años más tarde, Goodwin y Vermoten presentan dos casos de múltiples nódulos fibrosos de la túnica vaginal (3). Parveen y cols., revisa la literatura desde 1900 a 1992 encontrando 17 casos que cumplen criterios estrictos de tumor fibroso de la túnica vaginal (1).

Con las técnicas de microscopia electrónica y los estudios inmunohistoquímicos, podemos decir que esta lesión consiste en una proliferación mesenquimal fibroblástica vascularizada. Macroscópicamente se presenta como un nódulo único o múltiple bien circunscrito, encapsulado, independiente del parénquima testicular, que puede originarse tanto en el epidídimo como el cordón espermático o en estructuras escrotales adyacentes, suele ser de superficie firme y lisa, redondeado $u$ oval y su tamaño puede variar. Microscópicamente existen diversos patrones histológicos; generalmente son paucicelulares con escasas mitosis (menos de una por 10 campos de gran aumento), compuestos por una proliferación fibroblástica y focos inflamatorios en mayor o menor grado. La inmunohistoquimia demuestra positividad para vimentina, factor VIII en las células endoteliales, y frente al CD34 $(1,4,5)$.

No se ha descrito en ningún caso un comportamiento agresivo ni aparición de metástasis a distancia (6). Si ha habido opiniones discutidas acerca de su origen inflamatorio o neoplásico. Volviendo a su etiopatogenia, muchos autores los han considerado procesos reactivos y no neoplásicos aún denominándolos como fibromas $(1,4$,$) . Pero tan solo en alrededor del 30 \%$ de los pacientes se encuentra un antecedente de traumatismo u orquiepididimitis. Mostofi y Price utilizaban el término de pseudotumor fibroso, que es uno de los más empleados, por entender la lesión como una respuesta inflamatoria con proliferación fibrosa, opinión compartida por otros $(2,7)$. Por otro lado, Goodwin y Polsky distinguían los fibromas de las lesiones inflamatorias, las periorquitis proliferativas crónicas, a pesar de parecer similares histológicamente $(2,7,8)$. Goodwin reserva la denominación de periorquitis crónica, a la verdadera inflamación con engrosamiento de las túnicas que rodean al teste. Mientras que los tumores fibrosos de la túnica vaginal independientes del testículo, y que son una entidad clínica específica, aún muchas veces indistinguibles microscópicamente de la inflamación pura con engrosamiento de túnicas, se denominarían fibromas de la túnica vaginal o tumores fibrosos (2).

Según los antecedentes y las características anatomopatológicas del caso que hemos presentado, la idea de Goodwin nos parece acertada. En definitiva la inflamación crónica con reacción fibrosa y la proliferación fibrosa neoplásica, pueden acabar constituyendo una única entidad patológica, presentando en cada caso un desencadenante diferente. Tratándose todos estos apelativos de sinónimos de una misma lesión benigna.

El interés clínico que presenta esta entidad patológica es la clara ausencia de un comportamiento agresivo y potencial metastático. Constituye el tumor paratesticular más frecuente por detrás del tumor adenomatoide. A diferencia de las lesiones testiculares, 95\% de estas malignas, las lesiones paratesticulares suelen ser benignas siendo la ecografía y la exploración quirúrgica con biopsia peroperatoria determinantes para un adecuado manejo evitando la orquiectomía radical en una lesión benigna $(6,9,10)$. El tratamiento de elección es la exéresis de la túnica vaginal para poder quitar los tumores. En ocasiones si los nódulos fibrosos son numerosos sobre el epidídimo, puede ser imposible disecar la túnica vaginal del epidídimo incluyendo parte del epidídimo en la exéresis de la pieza (3).

\section{CONCLUSIONES}

A pesar de su extremada rareza, y lo poco conocida que es esta lesión por parte de los urólogos, alrededor de dos tercios de todos los pseudotumores fibrosos del área genital ocurren en las túnicas testiculares, por ello ante la aparición de un tumor fibroso en el escroto se debe realizar el diagnóstico diferencial con el tumor testicular, tumor del cordón espermático y tumor de las túnicas testiculares.

Los tumores fibrosos solitarios representan neoplasias mesenquimales que se caracterizan por ser ubicuas sin limitarse a las superficies serosas principales, pleura y peritoneo. El mecanismo patogénico, causa y origen celular de estos tumores son desconocidos. Su etiopatogenia ha sido ampliamente discutida y sigue siendo 
confusa. La identificación de estas lesiones es importante para evitar diagnósticos erróneos con otras lesiones de comportamiento agresivo localizadas en tejidos blandos. El pseudotumor fibroso de la túnica vaginal es una entidad clínica y patológica específica, aunque rara, puede ser reconocida intraoperatoriamente si se piensa en ella. No todas las masas escrotales se deben considerar malignas. Dado la falta de consenso en la literatura se entienden como sinónimos los términos que se han venido empleando para referirse a estas proliferaciones fibrosas de la túnica vaginal.

\section{BIBLIOGRAFIA y LECTURAS RECOMENDADAS ( ${ }^{*}$ lectura de interés $y^{* *}$ lectura fundamental)}

**1. PARVEEN, T.; FLEISCHMANN, J.; PETRELLI, M.: "Benign fibrous tumor of the tunica vaginalis testis". Arch. Pathol. Lab. Med., 116: 277, 1992.

**2. GOODWIN, W.E.: "Multiple, benign, fibrous tumors of tunica vaginalis testis". J. Urol., 56: 438, 1946.

3. LEWIS, H.Y.; PIERCE, J.M.: "Multiple fibromas of the tunica vaginalis". J. Urol., 87: 142, 1962.

4. JONES, M.A.; YOUNG, R.H.; SCULLY, R.E.: "Benign fibromatous tumors of the testis and paratesticular region: a report of 9 cases with a proposed classification of fibromatous tumors and tumor-like lesions". Am. J. Surg. Pathol., 21: 296, 1997.

*5. GOODLAD, J.R.; FLETCHER, C.D.: "Solitary fibrous tumor arising at unusual sites: analysis of a series". Histopathology, 19: 515, 1991.

*6. GARCIA, M.; BUSTOS, C.; DOMINGUEZ, E. y cols.: "A fibrous pseudotumor of the tunica vaginalis testis. A report of a new case and review of the literature". Actas Urol. Esp., 23: 68, 1999.

7. SARLIS, I.; YAKOYMAKIS, S.; REBELAKOS, A.G.: "Fibrous pseudotumor of the scrotum". J. Urol., 124: 742, 1980.

8. GORDON, W.S.: "Pseudotumor of testicular tunic". J. Urol., 118: 340, 1977.

*9. TOBIAS-MACHADO, M.; CORREA, A.; HELOISA, L. y cols.: "Fibrous pseudotumor of tunica vaginalis and epididymis". Urology, 56: 670, 2000.

10. RUBENSTEIN, R.A.; DOGRA, V.S.; SEFTEL, A.D. y cols.: "Benign intrascrotal lesions". J. Urol., 171: 1765, 2004.

\section{Casos Clínicos}

Arch. Esp. Urol., 59, 2 (189-192), 2006

\section{NEUROSÍFILIS Y DISFUNCIÓN VESICAL.}

Antonio Jalón Monzón, Alfonso San Martín Blanco,

Jorge García Rodríguez, José Luis Martín Benito, Oscar Rodríguez Faba, Roberto Carlos González Álvarez, Miguel Álvarez Múgica, Laura Rodríguez Robles y Francisco Javier Regadera Sejas.

Servicio de Urología. Hospital Universitario Central de Asturias. Oviedo. Asturias. España.

Resumen.- OBJETIVO: La sifilis es una enfermedad sistémica que cursa en estadios clínicos sucesivos. La afectación del sistema nervioso central y de la médula espinal en fases tardías puede provocar disfunción vesical. Presentamos un caso de neurosifilis con hiperreflexia asociada.

MÉTODO: Varón de 51 años diagnosticado de neurosífilis que consultó por trastornos miccionales objetivando en el estudio urodinámico un detrusor hiperactivo de causa neurogénica.

CONCLUSIONES: En el diagnóstico diferencial de vejiga neurógena en pacientes que presentan síntomas psiquiátricos y neurológicos debería ser considerada la neurosífilis. El diagnóstico etiológico es por examen del líquido cefalorraquídeo y el diagnóstico de su disfunción vesical mediante estudio urodinámico.

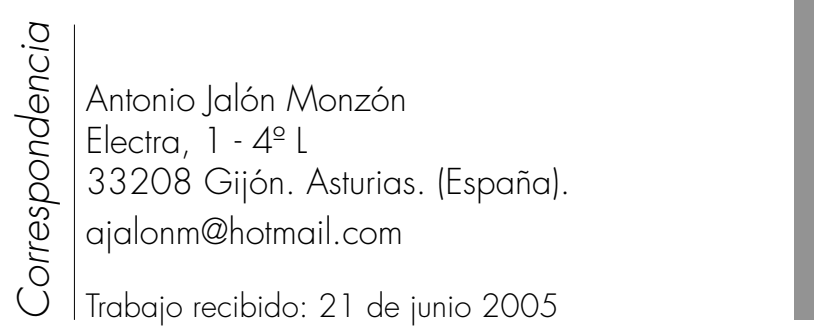

